ULB-TH/08-42

\title{
Duality and integrability: Electromagnetism, linearized gravity and massless higher spin gauge fields as bi-Hamiltonian systems
}

\author{
Glenn Barnich ${ }^{a}$ and Cédric Troessaert ${ }^{b}$ \\ Physique Théorique et Mathématique, Université Libre de Bruxelles \\ and \\ International Solvay Institutes, \\ Campus Plaine C.P. 231, B-1050 Bruxelles, Belgium
}

\begin{abstract}
In the reduced phase space of electromagnetism, the generator of duality rotations in the usual Poisson bracket is shown to generate Maxwell's equations in a second, much simpler Poisson bracket. This gives rise to a hierarchy of bi-Hamiltonian evolution equations in the standard way. The result can be extended to linearized Yang-Mills theory, linearized gravity and massless higher spin gauge fields.
\end{abstract}

${ }^{a}$ Research Director of the Fund for Scientific Research-FNRS (Belgium).

${ }^{b}$ Research Fellow of the Fund for Scientific Research-FNRS (Belgium). 


\section{Introduction}

A cornerstone of soliton theory is the discovery that the evolution equations are Hamiltonian systems [1, 2]. In this context, the occurrence of hierarchies of evolution equations sharing the same infinite set of conservation laws can be understood as a consequence of the existence of a second, compatible Hamiltonian structure giving rise to the same evolution equations [3, 4].

The equations of motion associated to the theories for the known fundamental forces of nature, electromagnetism, Yang-Mills theories and gravitation, are variational and thus Hamiltonian. This is no coincidence, since these theories are fundamentally quantum, at least the first three of them, and only for variational theories quantization is sufficiently well understood.

In order to have Poincaré invariance, respectively diffeomorphism invariance, manifestly realized, most modern investigations of these equations are carried out in the Lagrangian framework. This could be the reason why the bi-Hamiltonian structure underlying these equations and discussed below has hitherto remained unnoticed.

At the heart of our analysis is an important exception to this paradigm, namely the question whether the duality invariance of the four dimensional Maxwell or linearized gravity equations admits a canonical generator. This question has been answered to the affirmative in the reduced phase space of these theories and generalized to massless higher spin gauge fields [5, 6, 7].

We will show in this letter that the reduced phase space formulation of massless higher spin gauge fields is bi-Hamiltonian. The second Poisson bracket on reduced phase space turns out to be more natural than the one induced from the covariant variational principle, while the generator for duality rotations plays the role of the second Hamiltonian.

We will start with the most familiar case of Maxwell's equations, for which all details will be given. This result trivially generalizes to Yang-Mills theory with an invariant, non degenerate metric, linearized around a zero potential by decorating the expressions obtained in the electromagnetic case with an additional Lie algebra index.

The reduced phase space of linearized gravity around flat spacetime consists of two symmetric transverse and traceless potentials [8]. The entire analysis of the electromagnetic case then carries over in a straightforward way by taking care of the additional spatial index. The same goes for massless gauge fields of spin higher than 2 .

Several generalizations and extensions are suggested by the result reported here. A first exercise consists in studying the consequences for symmetries and conservation laws of both the Maxwell and the higher spin equations and compare them to known results (see e.g. [9, 10, 11] and references therein). Another obvious question is to investigate more general backgrounds. For instance, the generalization to massless spins propa- 
gating on (anti-) de Sitter spaces instead of Minkowski spacetime and the inclusion of fermionic gauge fields should be straightforward. In Yang-Mills theories (anti) self-dual backgrounds could be promising in view of their close connection to integrable systems.

The most important problem is however the inclusion of interactions. When comparing to the Korteweg-de Vries equation for instance, the present work corresponds to the bi-Hamiltonian structure for the linearized equation. The question is then to find interactions that preserve this structure. This will most probably not work for the standard interactions, as it is known that duality invariance does not survive the standard YangMills type coupling, nor the extension from linearized to full gravity [5, 12]. A promising candidate for an interacting model in which duality invariance does survive is Born-Infeld theory 1 . We plan to address at least some of these questions elsewhere.

\section{Electromagnetism}

The first order Hamiltonian action for free electromagnetism is

$$
\begin{aligned}
S\left[A_{i}, E^{i}, A_{0}\right] & =\int d t\left[\int d^{3} x\left(-E^{i} \partial_{0} A_{i}-A_{0} \partial_{i} E^{i}\right)-H\right], \\
H & =\frac{1}{2} \int d^{3} x\left[E^{i} E_{i}+(\mathcal{O} A)^{i}(\mathcal{O} A)_{i}\right] .
\end{aligned}
$$

Here, $A_{i}$ is the vector potential, $E^{i}$ the electric field and $A_{0}$ the Lagrange multiplier for the Gauss constraint. We use $x^{0}=t$ and $\mathcal{O}$ is the curl, $(\mathcal{O} A)^{i}=\epsilon^{i j k} \partial_{j} A_{k}$. Unless otherwise specified, the summation convention is used, Roman indices $i, j, k \ldots$ take values from 1 to 3 , are raised and lowered with the Kronecker delta, while $\epsilon_{i j k}$ is completely skewsymmetric with $\epsilon_{123}=1$.

The decomposition of $A_{i}, E^{i}$ into transverse and longitudinal vectors $A_{i}=A_{i}^{T}+$ $A_{i}^{L}, E^{i}=E^{T i}+E^{L i}$ allows one to eliminate the pure gauge degrees of freedom $A_{i}^{L}, E^{i L}$ associated with the Gauss constraint $\partial_{i} E^{i}=0$. By introducing a second vector potential $Z_{i}$ such that $E^{i T}=(\mathcal{O} Z)^{i}$ the reduced phase space action has been shown to be invariant under duality rotations [5]. This is most transparent in the double potential notation [13] where $A_{i}^{a} \equiv\left(A_{i}, Z_{i}\right)$. Roman indices $a, b, c \ldots$ take values from 1 to 2 , are raised and lowered with the Kronecker delta, while $\epsilon_{a b}$ is completely skew-symmetric with $\epsilon_{12}=1$. In this notation, the reduced phase space action can be written as

$$
\begin{gathered}
S^{R}\left[A_{i}^{T a}\right]=\int d t\left[\int d^{3} x \frac{1}{2} \epsilon_{a b}\left(\mathcal{O} A^{T a}\right)^{i} \partial_{0} A_{i}^{T b}-H_{1}\right], \\
H_{1}=\frac{1}{2} \int d^{3} x\left(\mathcal{O} A^{T a}\right)_{i}\left(\mathcal{O} A_{a}^{T}\right)^{i}=-\frac{1}{2} \int d^{3} x A^{T a i} \Delta A_{a i}^{T},
\end{gathered}
$$

\footnotetext{
${ }^{1}$ The authors thank X. Bekaert for pointing this out.
} 
where in the second expression for the Hamiltonian, we have used that $\mathcal{O}$ is "self-adjoint",

$$
\int d^{3} x g_{i}(\mathcal{O} f)^{i}=\int d^{3} x(\mathcal{O} g)_{i} f^{i}
$$

and

$$
\left(\mathcal{O}^{2} f^{T}\right)^{i}=-\Delta f^{T i}
$$

with $\Delta=\partial_{i} \partial^{i}$ the Laplacian in flat space. The standard Poisson bracket determined by the kinetic term is

$$
\left\{A_{i}^{T a}(x), A^{T b j}(y)\right\}_{1}=\epsilon^{a b} \Delta^{-1} \epsilon^{j k l} \partial_{k}^{y} \delta_{i l}^{T(3)}(x-y)=\epsilon^{a b} \Delta^{-1}\left(\mathcal{O}^{y} \delta_{i}^{T(3)}(x-y)\right)^{j},
$$

where $\delta_{i j}^{T(3)}(x-y)$ is the transverse delta function, see e.g. [14] section $A_{1}$.2. In vacuum, Maxwell's equations for the physical degrees of freedom read

$$
\partial_{0} A^{T a i}(x)=\left\{A^{T a i}(x), H_{1}\right\}_{1}=-\epsilon^{a b}\left(\mathcal{O} A_{b}^{T}\right)^{i}(x),
$$

while the generator for duality rotations is

$$
H_{0}=-\frac{1}{2} \int d^{3} x A_{T}^{a i}\left(\mathcal{O} A_{a}^{T}\right)_{i}, \quad\left\{H_{0}, H_{1}\right\}_{1}=0 .
$$

Remarkably, the duality generator is simply an $S O(2)$ Chern-Simons action [15].

When presented in this way, the second Hamiltonian structure is obvious and a lot simpler than the one induced from the covariant action principle. Indeed, a natural Poisson bracket on reduced phase space is simply

$$
\left\{A_{i}^{T a}(x), A_{j}^{T b}(y)\right\}_{0}=\epsilon^{a b} \delta_{i j}^{T(3)}(x-y),
$$

in terms of which the duality generator is the new Hamiltonian for Maxwell's equations,

$$
\left\{A^{T a i}(x), H_{1}\right\}_{1}=\left\{A^{T a i}(x), H_{0}\right\}_{0} .
$$

This is our main result.

At this stage, one can pause and ask whether electromagnetism and its quantization should not be based on this new Hamiltonian structure. A good reason to favour the old, more complicated structure is that, by construction, the Poincaré and conformal symmetries admit canonical generators for the old structure, while not all of them do for the new one. We plan to return to this question in detail elsewhere.

The reason why we are interested in the new structure is not so much in order to use it as an alternative for quantization, but because of what it tells us about Maxwell's equations themselves. For instance, using the bi-Hamiltonian structure, there is a standard way to generate an infinite set of generators that commute with the Hamiltonian and are 
in involution, which we now briefly describe (see e.g. [16], chapter 7.3 for an elementary introduction).

The recursion operator is obtained by contracting the new Poisson structure with the inverse of the original one,

$$
\mathcal{R}^{a i}{ }_{b j}=-\delta_{b}^{a}(\mathcal{O})_{j}^{i} .
$$

Consider, for $p \geqslant 1, K_{p}^{T a i}=(-)^{p} \epsilon^{a b}\left(\mathcal{O}^{p} A_{b}^{T}\right)^{i}$, or equivalently,

$$
K_{2 n+1}^{T a i}(x)=(-)^{n+1} \epsilon^{a b} \Delta^{n}\left(\mathcal{O} A_{b}^{T}\right)^{i}(x), \quad K_{2 n+2}^{T a i}(x)=(-)^{n+1} \epsilon^{a b} \Delta^{n+1} A_{b}^{T i}(x),
$$

for $n \geqslant 0$. The evolution equations of the hierarchy

$$
\partial_{0} A^{T a i}(x)=K_{p}^{T a i}(x), \quad \forall p \geqslant 1,
$$

are also bi-Hamiltonian,

$$
K_{p}^{T a i}(x)=\left\{A^{T a i}(x), H_{p}\right\}_{1}=\left\{A^{T a i}(x), H_{p-1}\right\}_{0},
$$

where $H_{p-1}=\frac{(-)^{p}}{2} \int d^{3} x A_{i}^{T a}\left(\mathcal{O}^{p} A^{T}\right)_{i}$,

$$
H_{2 n}=\frac{(-)^{n+1}}{2} \int d^{3} x A^{T a i} \Delta^{n}\left(\mathcal{O} A_{a}^{T}\right)_{i}, H_{2 n+1}=\frac{(-)^{n+1}}{2} \int d^{3} x A_{i}^{T a} \Delta^{n+1} A_{a i}^{T} .
$$

with Hamiltonians that are in involution,

$$
\left\{H_{n}, H_{m}\right\}_{1}=0=\left\{H_{n}, H_{m}\right\}_{0}, \quad \forall n, m \geqslant 0 .
$$

\section{Linearized gravity}

The Hamiltonian formulation of general relativity linearized around flat spacetime is based on the first order action principle [8]

$$
\begin{gathered}
S_{P F}\left[h_{m n}, \pi^{m n}, n_{m}, n\right]=\int d t\left[\int d^{3} x\left(\pi^{m n} \dot{h}_{m n}-n^{m} \mathcal{H}_{m}-n \mathcal{H}\right)-H_{P F}\right] \\
H_{P F}\left[h_{m n}, \pi^{m n}\right]=\int d^{3} x\left(\pi^{m n} \pi_{m n}-\frac{1}{2} \pi^{2}+\frac{1}{4} \partial^{r} h^{m n} \partial_{r} h_{m n}-\right. \\
\left.-\frac{1}{2} \partial_{m} h^{m n} \partial^{r} h_{r n}+\frac{1}{2} \partial^{m} h \partial^{n} h_{m n}-\frac{1}{4} \partial^{m} h \partial_{m} h\right) \\
\mathcal{H}_{m}=-2 \partial^{n} \pi_{m n}, \quad \mathcal{H}_{\perp}=\Delta h-\partial^{m} \partial^{n} h_{m n} .
\end{gathered}
$$

Here, $h=h^{m}{ }_{m}, \pi=\pi^{m}{ }_{m}$ and the linearized 4 metric is reconstructed using $h_{00}=-2 n$ and $h_{0 i}=n_{i}$.

Duality invariance of massless spin 2 fields has been uncovered by introducing suitable potentials for the linearized three metric and its momentum [6]. We will use below properties of these potentials discussed in more details in [17, 7, 18]. 
Symmetric rank two tensors $\phi_{m n}$ decompose as

$$
\begin{aligned}
\phi_{m n} & =\phi_{m n}^{T T}+\phi_{m n}^{T}+\phi_{m n}^{L} \\
\phi_{m n}^{L} & =\partial_{m} \psi_{n}+\partial_{n} \psi_{m} \\
\phi_{m n}^{T} & =\frac{1}{2}\left(\delta_{m n} \Delta-\partial_{m} \partial_{n}\right) \psi^{T} .
\end{aligned}
$$

The tensor $\phi_{m n}^{T}$ contains the trace of the transverse part of $\phi_{m n}$ and only one independent component. Three independent components are encoded in the longitudinal part $\phi_{m n}^{L}$, while the transverse-traceless part $\phi_{m n}^{T T}$ containing two independent components is defined as the remainder,

$$
\phi_{m n}^{T T}=\phi_{m n}-\phi_{m n}^{T}-\phi_{m n}^{L}
$$

The elements of the decomposition are orthogonal under integration if boundary terms can be neglected,

$$
\int d^{3} x \phi^{m n} \varphi_{m n}=\int d^{3} x\left(\phi^{T T m n} \varphi_{m n}^{T T}+\phi^{L m n} \varphi_{m n}^{L}+\phi^{T m n} \varphi_{m n}^{T}\right) .
$$

The generalized curl [7, 12] is defined through,

$$
(\mathcal{O} \phi)_{m n}=\frac{1}{2}\left(\epsilon_{m p q} \partial^{p} \phi_{n}^{q}+\epsilon_{n p q} \partial^{p} \phi_{m}^{q}\right)
$$

It satisfies

$$
\left(\mathcal{O}\left(\mathcal{O} \phi^{T T}\right)\right)_{m n}=-\Delta \phi_{m n}^{T T}
$$

and is self-adjoint,

$$
\int d^{3} x\left(\mathcal{O} \phi^{T T}\right)^{m n} \varphi_{m n}^{T T}=\int d^{3} x \phi^{T T m n}\left(\mathcal{O} \varphi^{T T}\right)_{m n} .
$$

The reduced phase space action for linearized gravity is

$$
\begin{aligned}
S^{R} & =\int d t\left[\int d^{3} x \pi^{T T m n} \partial_{0} h_{m n}^{T T}-H_{1}\right] \\
H_{1} & =\int d^{3} x\left(\pi_{T T}^{m n} \pi_{m n}^{T T}+\frac{1}{4} \partial_{r} h_{m n}^{T T} \partial^{r} h_{T T}^{m n}\right) .
\end{aligned}
$$

In the reduced phase space, one can make the change of variables,

$$
h_{m n}^{T T}=2\left(\mathcal{O} H^{1 T T}\right)_{m n}, \quad \pi_{m n}^{T T}=-\Delta H_{m n}^{2 T T} .
$$

so that the reduced phase space action becomes

$$
\begin{gathered}
S^{R}\left[H_{m n}^{T T a}\right]=\int d t\left[\int d^{3} x \epsilon_{a b} \Delta\left(\mathcal{O} H^{T T a}\right)^{m n} \partial_{0} H_{m n}^{T T b}-H_{1}\right] \\
H_{1}=\int d^{3} x\left(H^{T T a m n} \Delta^{2} H_{a m n}^{T T}\right) .
\end{gathered}
$$


The standard Poisson bracket determined by the kinetic term is

$$
\left\{H_{m n}^{T T a}(x), H^{T T b k l}(y)\right\}_{1}=\frac{1}{2} \epsilon^{a b} \Delta^{-2}\left(\mathcal{O}^{y} \delta_{m n}^{(3) T T}(x-y)\right)^{k l}
$$

where $\delta_{m n}^{(3) T T k l}(x-y)$ denotes the projector on the transverse-traceless part of a symmetric rank two tensor. The duality generator is

$$
D=-\int d^{3} x H^{T T a m n} \Delta\left(\mathcal{O} H_{a}^{T T}\right)_{m n}, \quad\left\{D, H_{1}\right\}_{1}=0 .
$$

The analogy with the spin 1 case can be made perfect by the change of variables,

$$
H_{m n}^{1 T T}=\frac{1}{\sqrt{2}} \Delta^{-1}\left(\mathcal{O} A^{2 T T}\right)_{m n}, \quad H_{m n}^{2 T T}=\frac{1}{\sqrt{2}} \Delta^{-1}\left(\mathcal{O} A^{1 T T}\right)_{m n} .
$$

in terms of which

$$
\begin{gathered}
H_{1}=\frac{1}{2} \int d^{3} x\left(\left(\mathcal{O} A^{T T a}\right)^{m n}\left(\mathcal{O} A_{a}^{T T}\right)_{m n}\right)=-\frac{1}{2} \int d^{3} x\left(A^{T T a m n} \Delta A_{a m n}^{T T}\right), \\
\left\{A_{m n}^{T T a}(x), A^{T T b k l}(y)\right\}_{1}=\epsilon^{a b} \Delta^{-1}\left(\mathcal{O}^{y} \delta_{m n}^{(3) T T}(x-y)\right)^{k l} \\
H_{0}=-D=-\frac{1}{2} \int d^{3} x A^{T T a m n}\left(\mathcal{O} A_{a}^{T T}\right)_{m n}
\end{gathered}
$$

All formulae of section 2 below equation (2.9) now generalize in a straightforward way to massless spin 2 fields by replacing $T$ (transverse) by $T T$ (transverse-traceless) and contracting over the additional spatial index.

\section{Massless higher spin gauge fields}

The extension of these results to massless higher spin gauge fields [19] (see also [20]) follows directly from the observation that the Hamiltonian reduced phase space formulation of these theories merely involves additional spatial indices [7], so that all above results generalize in a straightforward way.

This can be seen for instance by starting from the approach inspired from string field theory, where the Lagrangian action for massless higher spin gauge fields is written as the mean value of the BRST charge for a suitable first quantized particle model [21, 22, 23] (see also [24, 25] for further developments). In this framework the reduction of the action to the light-cone gauge corresponds to the elimination of BRST quartets composed of ghost and light-cone oscillators (see e.g.[26, 27]). In exactly the same way, the ghost, temporal and longitudinal oscillators form quartets that can be eliminated to yield the Lagrangian gauge fixed action for a massless field of spin $s \geqslant 1$,

$$
S_{L}\left[\phi_{i_{1} \ldots i_{s}}^{T T}\right]=-\frac{1}{2} \int d^{4} x \partial_{\mu} \phi_{i_{1} \ldots i_{s}}^{T T} \partial^{\mu} \phi^{T T i_{1} \ldots i_{s}}
$$


where the field $\phi_{i_{1} \ldots i_{s}}^{T T}$ is real, completely symmetric, traceless and transverse,

$$
\phi_{i_{1} \ldots i_{s}}^{T T}=\phi_{\left(i_{1} \ldots i_{s}\right)}^{T T}, \phi_{i i_{3} \ldots i_{s}}^{T T i}=0, \partial^{i} \phi_{i i_{2} \ldots i_{s}}^{T T}=0 .
$$

The Hamiltonian formulation is direct, the momenta being $\pi_{i_{1} \ldots i_{s}}^{T T}=\partial_{0} \phi_{i_{1} \ldots i_{s}}^{T T}$.

Consider then the Fock space defined by $\left[a^{i}, a_{j}^{\dagger}\right]=\delta_{j}^{i}, a_{i}|0\rangle=0$, the number operator $N=a_{i}^{\dagger} a^{i}$, the "string field" $\phi_{s}^{T T}(x)=\frac{1}{\sqrt{s !}} a_{i_{1}}^{\dagger} \ldots a_{i_{s}}^{\dagger}|0\rangle \phi_{i_{1} \ldots i_{s}}^{T T}(x)$ and the inner product

$$
\left\langle\phi_{s}^{T T}, \psi_{s}^{T T}\right\rangle=\int d^{3} x\left\langle\phi_{s}^{T T}, \psi_{s}^{T T}\right\rangle_{F}=\int d^{3} x \phi_{i_{1} \ldots i_{s}}^{T T} \psi^{T T i_{1} \ldots i_{s}} .
$$

With this inner product, the generalized curl [7]

$$
\mathcal{O}=\frac{1}{N} \epsilon^{i j k} a_{i}^{\dagger} \partial_{j} a_{k}
$$

is again self-adjoint. Furthermore, it squares to $-\Delta$ inside the inner product involving transverse-traceless fields,

$$
\begin{aligned}
\mathcal{O}^{2} & =\frac{1}{N^{2}}\left[-\Delta N^{2}+\left(\partial \cdot a^{\dagger}\right)(\partial \cdot a)+\left(a^{\dagger} \cdot a^{\dagger}\right) \Delta(a \cdot a)+2\left(\partial \cdot a^{\dagger}\right) N(\partial \cdot a)\right. \\
& \left.-\left(\partial \cdot a^{\dagger}\right)^{2}(a \cdot a)-\left(a^{\dagger} \cdot a^{\dagger}\right)(\partial \cdot a)^{2}\right] \Longrightarrow\left\langle\phi_{s}^{T T}, \mathcal{O}^{2} \psi_{s}^{T T}\right\rangle=-\left\langle\phi_{s}^{T T}, \Delta \psi_{s}^{T T}\right\rangle .
\end{aligned}
$$

The change of variables making duality invariance transparent is

$$
\phi_{i_{1} \ldots i_{s}}^{T T}=A_{i_{1} \ldots i_{s}}^{T T 1}, \pi_{i_{1} \ldots i_{s}}^{T T}=-\left(\mathcal{O} A^{T T 2}\right)_{i_{1} \ldots i_{s}} .
$$

The first order reduced phase space action becomes

$$
\begin{gathered}
S^{R}\left[A^{T T a}\right]=\int d t\left[\int d^{3} x \frac{1}{2} \epsilon_{a b}\left(\mathcal{O} A^{T T a}\right)^{i_{1} \ldots i_{s}} \partial_{0} A_{i_{1} \ldots i_{s}}^{T T b}-H_{1}\right] \\
H_{1}=-\frac{1}{2} \int d^{3} x A_{i_{1} \ldots i_{s}}^{T T a} \Delta A_{a}^{T T i_{1} \ldots i_{s}} .
\end{gathered}
$$

Again, all formulae of section 2 below equation (2.8), including the one for the duality generator, suitably generalize by contracting over the additional spatial indices.

\section{Acknowledgements}

The authors thank M. Henneaux for a most useful discussion. This work is supported in part by a "Pôle d'Attraction Interuniversitaire" (Belgium), by IISN-Belgium, convention 4.4505.86, by the Fund for Scientific Research-FNRS (Belgium), and by the European Commission programme MRTN-CT-2004-005104, in which the authors are associated to V.U. Brussel. 


\section{References}

[1] C. S. Gardner, "Korteweg-de Vries Equation and Generalizations. IV. The Korteweg-de Vries Equation as a Hamiltonian System," Journal of Mathematical Physics 12 (1971), no. 8, 1548-1551.

[2] V. E. Zakharov and L. D. Faddeev, "Korteweg-de Vries equation: A completely integrable Hamiltonian system," Functional Analysis and Its Applications 5 (10, 1971) 280-287.

[3] F. Magri, "A simple model of the integrable Hamiltonian equation," Journal of Mathematical Physics 19 (1978), no. 5, 1156-1162.

[4] I. M. Gel'fand and I. Y. Dorfman, "Hamiltonian operators and algebraic structures related to them," Functional Analysis and Its Applications 13 (10, 1979) 248-262.

[5] S. Deser and C. Teitelboim, "Duality transformations of abelian and nonabelian gauge fields," Phys. Rev. D13 (1976) 1592-1597.

[6] M. Henneaux and C. Teitelboim, "Duality in linearized gravity," Phys. Rev. D71 (2005) 024018, gr-qc/0408101.

[7] S. Deser and D. Seminara, "Duality invariance of all free bosonic and fermionic gauge fields," Phys. Lett. B607 (2005) 317-319, hep-th/0411169.

[8] R. Arnowitt, S. Deser, and C. Misner, Gravitation, an Introduction to Current Research, ch. 7. The Dynamics of General Relativity, pp. 227-265. Wiley, New York, 1962.

[9] W. I. Fushchich and A. G. Nikitin, "The complete sets of conservation laws for the electromagnetic field," Journal of Physics A: Mathematical and General 25 (1992), no. 5, L231-L233.

[10] S. C. Anco and J. Pohjanpelto, "Conserved currents of massless fields of spin s 1/2," Proceedings: Mathematical, Physical and Engineering Sciences 459 (2003), no. 2033, 1215-1239.

[11] J. Pohjanpelto and S. Anco, "Generalized symmetries of massless free fields on Minkowski space," SIGMA 4 (2008) 17.

[12] S. Deser and D. Seminara, "Free spin 2 duality invariance cannot be extended to GR," Phys. Rev. D71 (2005) 081502, hep-th/0503030.

[13] J. H. Schwarz and A. Sen, "Duality symmetric actions," Nucl. Phys. B411 (1994) 35-63, hep-th/9304154. 
[14] C. Cohen-Tannoudji, J. Dupont-Roc, and G. Grynberg, Photons and Atoms: Introduction to Quantum Electrodynamics. Wiley-Interscience, 1989.

[15] S. Deser, M. Henneaux, and C. Teitelboim, "Electric - magnetic black hole duality," Phys. Rev. D55 (1997) 826-828, hep-th/9607182.

[16] P. Olver, Applications of Lie Groups to Differential Equations. Springer Verlag, New York, 2nd ed., 1993. 1st ed., 1986.

[17] S. Deser, "Covariant decomposition of symmetric tensors and the gravitational Cauchy problem.," Ann. Inst. Henri Poincare Sect. A, 7 (1967) 149-88.

[18] G. Barnich and C. Troessaert, "Manifest spin 2 duality with electric and magnetic sources," 0812.0552 .

[19] C. Fronsdal, "Massless fields with integer spin,” Phys. Rev. D18 (1978) 3624.

[20] B. de Wit and D. Z. Freedman, "Systematics of higher spin gauge fields," Phys. Rev. D21 (1980) 358.

[21] S. Ouvry and J. Stern, "Gauge fields of any spin and symmetry," Phys. Lett. B177 (1986) 335.

[22] A. K. H. Bengtsson, "A unified action for higher spin gauge bosons from covariant string theory,” Phys. Lett. B182 (1986) 321.

[23] M. Henneaux and C. Teitelboim, First and second quantized point particles of any spin, ch. 9, pp. 113-152. Quantum mechanics of fundamental systems 2, Centro de Estudios Científicos de Santiago. Plenum Press, 1987.

[24] A. Sagnotti and M. Tsulaia, "On higher spins and the tensionless limit of string theory,” Nucl. Phys. B682 (2004) 83-116, hep-th/0311257.

[25] G. Barnich, M. Grigoriev, A. Semikhatov, and I. Tipunin, "Parent field theory and unfolding in BRST first-quantized terms," Commun. Math. Phys. 260 (2005) 147-181, hep-th/0406192.

[26] W. Siegel, "Fields," hep-th/9912205.

[27] G. Barnich, G. Bonelli, and M. Grigoriev, "From BRST to light-cone description of higher spin gauge fields," Annals Univ. Craiova 15 part I (2005) 1-10, hep-th/0502232. 